\title{
Oncologic Panendoscopy: Description of an Optimized Procedure Based on Our Experience
}

\author{
Laurence Pincet $^{1}$ Cécile de Sandre ${ }^{1} \quad$ Florian J. W. Lang ${ }^{2} \quad$ Victor Colin ${ }^{20}$ \\ ${ }^{1}$ Department Department of Head and Neck Surgery, Centre \\ Hospitalier Universitaire Vaudois (CHUV), Lausanne, Switzerland \\ 2 Department of Head and Neck Surgery, Hôpital Cantonal \\ Fribourgeois, Fribourg, Switzerland

\begin{abstract}
Address for correspondence Dr. Laurence Pincet, MD, Department of Head and Neck Surgery, Centre Hospitalier Universitaire Vaudois (e-mail: laurence.pincet@chuv.ch).
\end{abstract} \\ (CHUV), Rue du Bugnon 46, Lausanne 1011, Switzerland
}

Int Arch Otorhinolaryngol 2022;26(1):e125-e131.

Abstract
Keywords
- squamous cell
carcinoma
- esophagoscopic
surgical procedure
- cancer screening test
- multiple primary
neoplasms
- synchronous
- synchronous
neoplasms

Introduction All patients with a new head and neck squamous cell carcinoma (HNSCC) undergo diagnostic panendoscopy as part of the screening for synchronous second primary tumors. It includes a pharyngolaryngoscopy (PLS), a tracheobronchoscopy and esophagoscopy, and a stomatoscopy. Rigid techniques are risky, with long learning curves.

Objective We propose a precise description of the panendoscopy protocol. We include an optimization of the PLS technique that completes the flexible esophagoscopy when rigid esophagoscopy isn't performed.

Methods The present retrospective observational study includes 122 consecutive patients with a new primary HNSCC who underwent traditional panendoscopy and the new PLS technique between January 2014 and December 2016. A two-step procedure using a Macintosh laryngoscope and a $30^{\circ}$ telescope first exposes panoramically the larynx, the upper trachea, and the oropharynx; then, in a second step, the hypopharynx is exposed down to the upper esophageal sphincter. Broncho-esophagoscopy is performed with a rigid and flexible scope.

Results In total, 6 (5\%) patients presented synchronous tumors ( 3 in the esophagus, 2 in the oral cavity, and 1 in the larynx 1$)$. Rigid endoscopy was complicated by $2(1,6 \%)$ dental lesions, and had to be completed with a flexible scope in 38 (33\%) cases for exposition reasons. The two-step PLS offered a wide-angle view of the larynx, upper trachea, and oro- and hypopharynx down to the sphincter of the upper esophagus. The procedure was easy, reliable, safe, repeatable, and effectively completed the flexible endoscopies.

Conclusion Rigid esophagoscopy remains a difficult procedure. Two-step PLS combined with flexible broncho-esophagoscopy offers good optical control. received

March 13, 2020

accepted

December 1, 2020

published online

August 9, 2021
DOI https://doi.org/ 10.1055/s-0041-1726049. ISSN 1809-9777.

\footnotetext{
(c) 2021. Fundação Otorrinolaringologia. All rights reserved.

This is an open access article published by Thieme under the terms of the Creative Commons Attribution-NonDerivative-NonCommercial-License, permitting copying and reproduction so long as the original work is given appropriate credit. Contents may not be used for commercial purposes, or adapted, remixed, transformed or built upon. (https://creativecommons.org/ licenses/by-nc-nd/4.0/)

Thieme Revinter Publicações Ltda., Rua do Matoso 170, Rio de Janeiro, RJ, CEP 20270-135, Brazil
} 


\section{Introduction with Objective}

In the literature, the incidence of second primary esophageal cancer in patients with head and neck squamous cell carcinoma (HNSCC) varies between $0 \%$ and $21.9 \% .^{1-3}$

Early neoplasia in the esophagus can go unnoticed on computed tomography (CT) scans or positron emission tomography coupled with tomodensitometry (PET-CT), ${ }^{4}$ so endoscopy is the gold-standard technique to diagnose preinvasive lesions. $^{5}$

Panendoscopy with a rigid tube is a well-known technique long performed by the Swiss school of bronchoesophagology since Savary. Since then, no guidelines guidelines have been developed on the use of rigid or flexible esophagoscopes. It rather depends on the center or the practitioner's habit. ${ }^{5}$ Rigid esophagoscopy, long favored by ear, nose and throat (ENT) surgeons, is gradually replaced by flexible esophagoscopy. ${ }^{6}$ They both enable the use narrow-band imaging (NBI) or the performance of a biopsy.

The advantage of rigid over flexible esophagoscopy is examining the hypopharynx and the upper esophagus sphincter's view. ${ }^{7}$ This examination is not possible in flexible esophagoscopy, and it has to be substituted by another pharyngolaryngoscopy (PLS) technique.

The objective of the present study is to propose a precise description of the panendoscopy protocol. We include an optimization of the PLS technique that completes the flexible esophagoscopy when rigid esophagoscopy isn't performed.

\section{Methods}

We conducted a monocentric retrospective observational study including all consecutive patients with a new HNSCC diagnosed from January 2014 to December 2016. All patients underwent a complete panendoscopy, including a PLS, a rigid tracheobronchoscopy, a rigid esophagoscopy, and a stomatoscopy. If needed, a flexible esophagoscopy was performed. Every step is described in the "Procedure" section of the present article. We used a Macintosh laryngoscope (KaWe KIRCHNER \& WILHELM (Germany) and $0^{\circ}, 30^{\circ}$, or $70^{\circ}$ short and long telescopes (Karl Storz SE \& Co. KG, Tuttlingen, Germany) for the PLS. Rigid tracheobronchoscopies were performed with rigid bronchoscopes (Storz) and matching $0^{\circ}, 30^{\circ}$ and $90^{\circ}$ telescopes; and the esophagoscopies were performed with a $0^{\circ}$ rigid esophagoscope (Storz) and a flexible esophagoscope (Olympus Medical Systems Corp., Shinjuku City, Tokyo, Japan). The biopsies were performed with Storz optical forceps or with Olympus reusable biopsy forceps during the flexible esophagoscopy.

Demographics of the patients and characteristics of their tumors were collected (-Table $\mathbf{1}$ ). The tumors were classified according to the International Classification of Diseases.

\section{Procedure}

All panendoscopies are performed in an operating room under general anesthesia. Rigid endoscopy requires curarization. Regardless of the patient's comorbidities, standard
Table 1 Demographics and characteristics of the tumors

\begin{tabular}{|c|c|}
\hline Patients & Characteristics \\
\hline - Age & 65.7 years (30-92 years) \\
\hline - Male/Female ratio & $18 / 1$ \\
\hline \multicolumn{2}{|l|}{ - Risk factors: } \\
\hline o Smoking & $105(86 \%)$ \\
\hline o Alcohol abuse & $72(59 \%)$ \\
\hline $\begin{array}{l}\text { o Human papillomavirus } \\
\text { infection }\end{array}$ & $15(12.3 \%)$ \\
\hline \multicolumn{2}{|l|}{ - First location } \\
\hline o Oral cavity & $35(28.7 \%)$ \\
\hline o Oropharynx & $35(28.7 \%)$ \\
\hline o Hypopharynx & $21(17.2 \%)$ \\
\hline o Larynx & 15 (12.3\%) \\
\hline o Esophagus & $2(1.6 \%)$ \\
\hline o Unknown & $14(11.5 \%)$ \\
\hline \multicolumn{2}{|l|}{ - First staging } \\
\hline \multicolumn{2}{|l|}{ o Tumor } \\
\hline $\boldsymbol{~ i s ~ ( i n ~ s i t u ) ~}$ & $4(3.3 \%)$ \\
\hline $\boldsymbol{\square} 1$ & $27(22.1 \%)$ \\
\hline $\mathbf{\square} 2$ & $24(19.7 \%)$ \\
\hline$\square 3$ & 25 (20.5\%) \\
\hline$\square 4$ & $26(21.3 \%)$ \\
\hline 口unknown primary & $14(11.5 \%)$ \\
\hline \multicolumn{2}{|l|}{ o Node } \\
\hline $\mathbf{0}$ & $53(43 \%)$ \\
\hline $\mathbf{\square} 1$ & $17(14 \%)$ \\
\hline$\square 2$ & $47(38 \%)$ \\
\hline$\square 3$ & $5(4 \%)$ \\
\hline Unknown & $1(0.8 \%)$ \\
\hline \multicolumn{2}{|l|}{ o Metastasis } \\
\hline$\square 0$ & $110(90.2 \%)$ \\
\hline $\mathbf{a} 1$ & $6(4.9 \%)$ \\
\hline Unknown & $4(3.3 \%)$ \\
\hline - Anterior radiotherapy & $2(1.6 \%)$ \\
\hline
\end{tabular}

monitoring (electrocardiography, blood pressure, oximetry, and capnography) is sufficient. The bispectral index is optional. A classic operating table is compatible with the procedure. During the whole procedure, close cooperation with the anesthesia team is crucial. The following steps take place successively:

\section{Direct Pharyngolaryngoscopy}

First of all, the anesthesiologist administers several minutes of preoxygenation. Then, depending on the pulmonary capacity of the patient, PLS is performed during one long apnea or several successive apneas. The anesthesiologists usually aim for $80 \%$ of expired oxygen before a new apnea. 

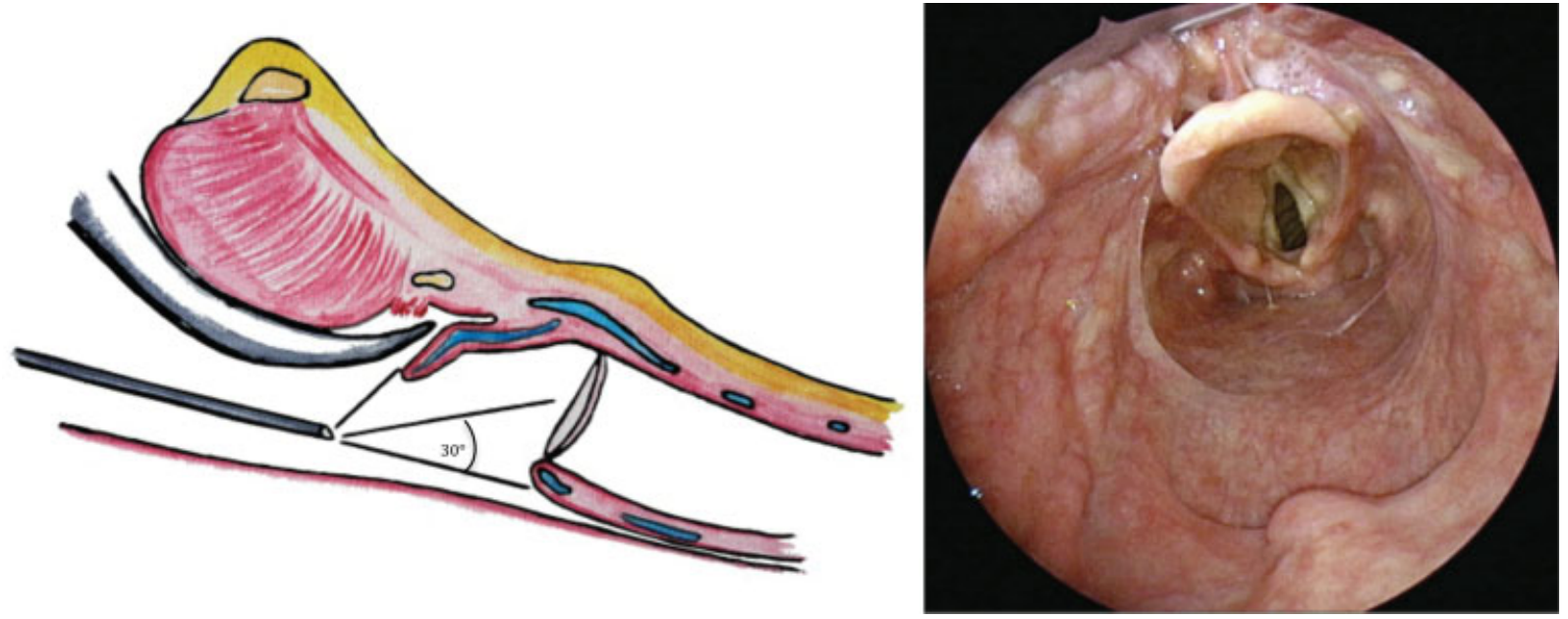

Fig. 1 First step of the panendoscopy.

\section{First Step (-Fig. 1)}

With the non-dominant hand maneuvering the Macintosh laryngoscope and the dominant hand holding the telescope, a close examination of the palate (soft and hard), tonsils, retromolar trigons, and posterior pharyngeal wall is performed. The blade is progressively positioned in the valleculae, exploring the epiglottis, the larynx, and the upper trachea. The $70^{\circ}$ telescope can be used for patients difficult to expose, especially to examine the anterior larynx or the laryngeal side of the epiglottis.

\section{Second Step (-Fig. 2)}

The blade is placed under the epiglottis, above the anterior commissure. Lifting enables charging of the laryngeal bloc and unfolding of the hypopharynx. The $30^{\circ}$ long telescope then explores the retro-arytenoid area, the pyriform sinus, and the sphincter of the upper esophagus (-Fig. 2). Photographs or videos can be made of the global view or close to a suspicious lesion. If needed, biopsies will be performed at the end of the procedure with the tube in place to avoid blood contamination and minimize blood aspiration.

\section{Rigid Tracheobronchoscopy}

A Teeth Guard is Placed for Protection of the Upper Teeth The rigid bronchoscope is gently introduced in the midline, with care not to damage the teeth. Lifting the epiglottis anteriorly with the tip of the bronchoscope enables progression and exposesure of the the laryngeal structures. An examination of the glottic area is repeated with a $90^{\circ}$ rotation of the bronchoscope on each side. As soon as the vocal cords are passed, the ventilation tube can be plugged into the bronchoscope to ventilate the patient. It is then pushed along the trachea up to the carina. Each left and right bronchial system is inspected with the angled telescopes, up to the subsegmental bronchi. Biopsies are performed with biopsy forceps if needed. Gentle removal of the bronchoscope with a $30^{\circ}$ optic turned upwards enables the
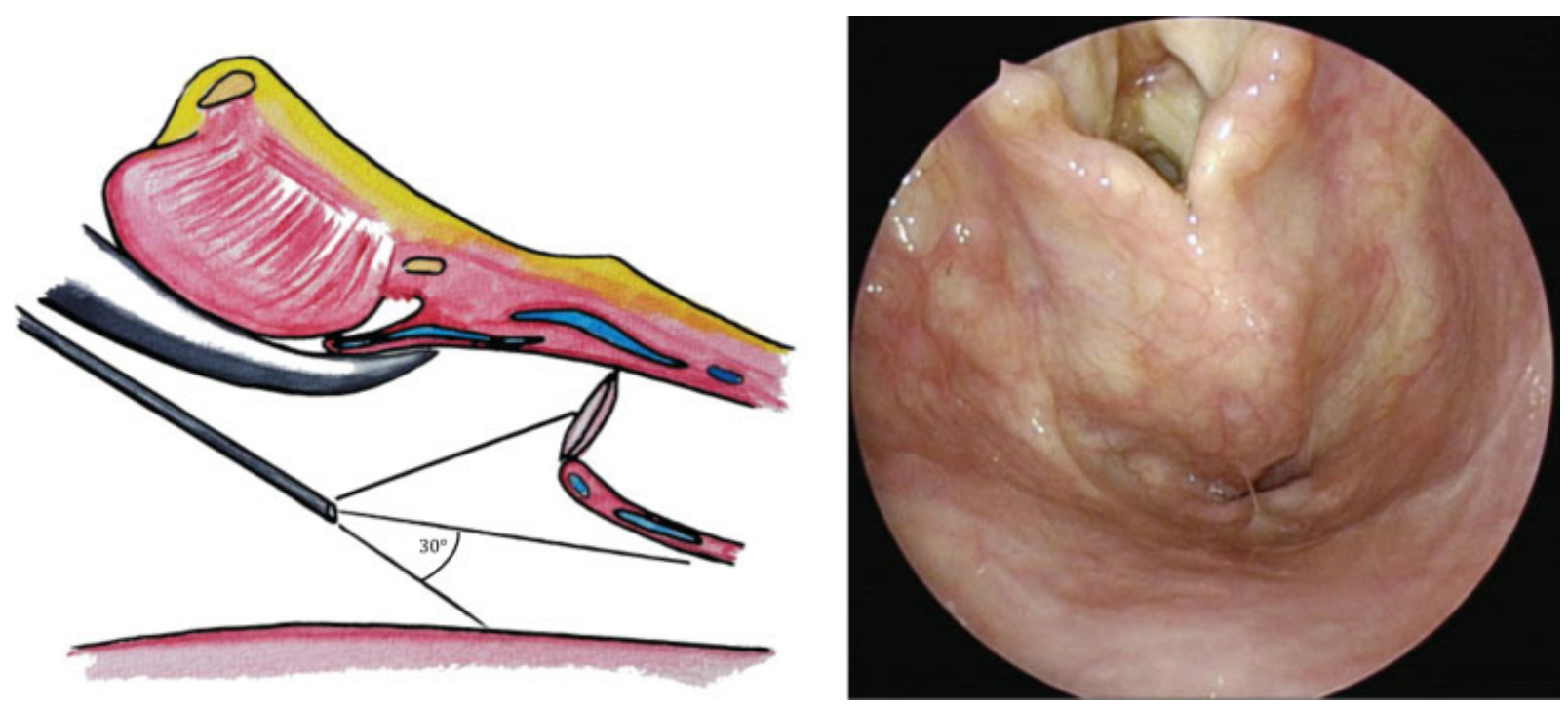

Fig. 2 Second step of the panendoscopy. 


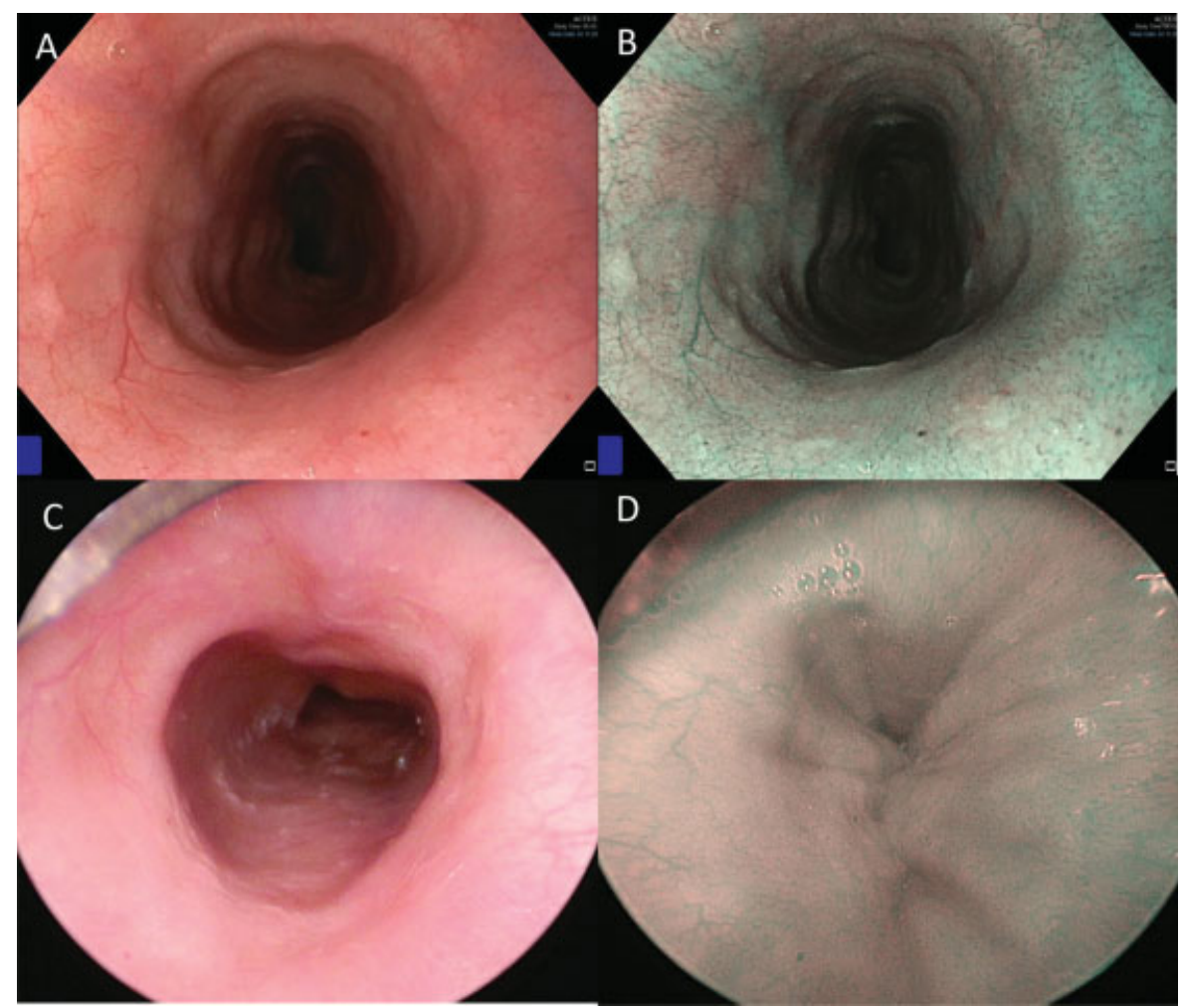

Fig. 3 Comparison between rigid and flexible esophagoscopy. (A, B): flexible esophagoscopy with the narrow-band imaging mode (B). (C,D) Rigid esophagoscopy with the narrow-band imaging mode (D).

reexamination of the subglottic area and anterior commissure, possibly with NBI.

The anesthesiologist intubates the patient, with the tube remaining in place until the end of the procedure. The tube is fixed on the left side to facilitate the esophagoscopy.

\section{Esophagoscopy (-Fig. 3)}

The patient is positioned with a cervical extension, and must be "pulled out" from the operating table until the shoulders are outside the table and are supported by the practitioner's knee, whose leg is bent at a right angle. An assistant can help keep the patient's head in position for more security. The esophagoscope is inserted via the right corner and floor of the mouth. Under optical control, the esophagoscope unfolds the piriform sinus and then progresses from the upper esophagus sphincter to the inferior sphincter. While the esophagoscope moves forward, cervical extension must increase so that the practitioner doesn't feel resistance. In the end, the cervical extension can be substantial, with the surgeon with one knee on the floor, and the patient's head supported by an assistant. Special care must be taken with the teeth during this procedure, with the esophagoscope always supported by the practitioner's left thumb.

The procedure can be completed with flexible esophagoscopy (see reasons below in the "Results" section), and a mouth guard is placed to protect the endoscope. The flexible esophagoscope is inserted through the mouth in the hypopharynx. Insufflations enable the opening of the upper sphincter and progression through the esophagus until the stomach.
Retrograde esophagoscopy (with either a rigid or flexible endoscope) is performed with NBI, leading to an improvement in the visualization of the intrapapillary capillary loops of the early squamous cell esophageal cancer. With the removal, the stomach is exsufflated, then careful examination of the upper sphincter is repeated.

-Fig. 3A and 3B illustrate the equivalence of image quality between the two techniques. The NBI mode can be used with both of them. Fig. $3 C$ shows the view during an insufflation; and Fig. 3D shows the esophagus collapsed between two insufflations.

\section{Stomatoscopy, Vital Staining:}

Vital staining is performed with toluidine blue after removing the excess of saliva with cotton swabs soaked with $1 \%$ acetic acid. The same two steps of the PLS are briefly repeated for the hypopharynx examination after the coloration, focusing on the suspicious lesions. A biopsy can be performed. Holding both the laryngoscope and telescope with the nondominant hand, the biopsy forceps is maneuvered with the dominant hand. Possibld suspicious lesions are palpated in the oral cavity.

The panendoscopy ends with cervical palpation looking for lymphadenopathy.

\section{Results}

We included 122 consecutive patients with a new primary HNSCC who underwent traditional panendoscopy and the optimized PLS technique (-Table 2). 
Table 2 Results of the panendoscopy

\begin{tabular}{|l|l|}
\hline Follow-up & 702 days (8-1,591 days) \\
\hline Overall survival & $89 \%$ (13 died) \\
\hline Procedure: & - \\
\hline - Duration of the pharyngolaryngoscopy & -4.6 minutes (2.7-11.5 minutes) \\
\hline - Global duration of the procedure & -72 minutes (37-105 minutes) \\
\hline Rigid esophagoscopy converted to flexible esophagoscopy & 38 cases (33\%) \\
\hline Synchronous second primary tumors: & 6 cases $(5 \%)$ \\
\hline - Esophagus & -3 cases $(2.5 \%)$ \\
\hline - Oral cavity & -2 cases $(1.6 \%)$ \\
\hline - Valleculae & -1 cases $(0.8 \%)$ \\
\hline Complications & 2 cases (1.6\%): dental lesions \\
\hline
\end{tabular}

In total, 13 patients died, 5 were followed up in other Swiss hospitals, and 1 (0,8\%) moved to Portugal. The whole panendoscopic procedure (including the PLS, bronchoscopy, esophagoscopy, and stomatoscopy) lasted, 72 minutes (range: 37 to 105 minutes) on average. In particular, the PSL itself lasted 4.6 minutes (range: 2.7 to 11.5 minutes) on average. There was no procedure failures and no complications. Rigid esophagoscopy was converted into flexible in 38 (33\%) cases: the main reason was a limited cervical extension (28 patients), associated or not with a narrow mouth opening (6 patients). Moreover, 13 (10,6\%) patients with bad oral hygiene had unstable upper teeth.

All procedures were photodocumented for tumor boards. Biopsies were performed during the consultation in 89 (73\%) cases, excluding patients who already had a diagnosis and no other suspicious lesion. All biopsies were of good quality and enabled a correct histological analysis. All patients underwent the procedure once; none needed reoperation.

In total, 6 (5\%) synchronous second primary tumors were found: ( 3 in the middle third of the esophagus, 2 in the oral cavity, and 1 in the valleculae). Within these six cases, four were associated with an oropharyngeal primary tumor one, with a hypopharyngeal primary tumor, and one, with an oral cavity primary tumor. All second primary tumors were identified with the PLS technique and flexible esophagoscopy.

A total of $2(1.6 \%)$ minor complications were reported (dental lesion) during the rigid esophagoscopy, none with the optimized PLS technique.

\section{Discussion}

We found synchronous second primary tumors in 6 (5\%) of our cases. Most of these patients had oropharyngeal primary tumors. The association between second primary tumor and oropharyngeal primary tumor has been reported in the literature. ${ }^{1-3,8}$

The optimized PLS technique was quick (medium procedure time of 4.6 minutes, raised for patients difficult to expose). There was no procedure failure, even for patients with voluminous tumors. The Macintosh laryngoscope and the $30^{\circ}$ telescope were maneuverable, providing a close and precise or panoramic view of a suspicious lesion, and facilitated the biopsies. Photodocumentation ( - Video 1 ) was used for every tumor board. Examination of the anterior commissure of the vocal cord is usually tricky. The $30^{\circ}$ and $70^{\circ}$ angulation facilitated the inspection and enabled the examination of every patient.
Video 1
Pharyngolaryngoscopy procedure. Online content including video sequences viewable at: https://s20.video-stream-hosting.de/tvg/ ejournal/10.1055-s-00025477/10-1055-s-0041- 1726049-iao-20-0023-v1_360p.mp4.

As a first choice, rigid esophagoscopy was performed for every patient, as it is the usual procedure. However, limited cervical extensions, narrow mouth opening, or fragile upper teeth made the exposition complicated, and rigid esophagoscopies had to be converted into flexible esophagoscopies to examine the lower part of the esophagus.

Rigid esophagoscopy added no sensitivity to the procedure: all primary or synchronous lesions were identified first with the PLS or later with flexible endoscopy. For exposition reasons, one-third was converted into flexible esophagoscopy, showing the difficulty involved in the rigid procedures.

The main limitation of the present study is its design: the retrospective monocentric model provides a low level of proof. The facility and quality of the pharyngolaryngeal examination is subjective. We had no PLS complications, but we could imagine lesions to the lips or teeth, and pharyngolaryngeal traumatism with the Macintosh blade.

Early neoplasia (cTis (in situ) and cT1) in the esophagus can go unnoticed on CT scans or PET-CT. ${ }^{4,9}$ In many ENT oncology centers, panendoscopy with rigid endoscopy has remained the gold standard for screening synchronous 
esophageal carcinomas in patients with a new HNSCC. Severe complications, such as iatrogenic esophageal perforation up to $2.6 \%{ }^{10,11}$, have a high mortality rate $(19 \%) .{ }^{11}$ Other minor complications frequently occur, such as esophageal wound, dental damage, or lesions to the oral mucosa. The complications are mostly related to exposition problems that make the progression of the esophagoscope difficult. The examination of the hypopharynx justifies the risk that is taken. But the gravity of such a risk has led many authors to reconsider the place of the rigid esophagoscopy in the staging of new HNSCCs.

The indication for esophagoscopy has been proposed to be limited to patients with a high risk of developing synchronous esophageal carcinomas. ${ }^{1}$ Koo et al. ${ }^{2}$ proposed a reevaluationof the role of panendoscopy in patients who do not drink alcohol or smoke. The location of primary HNSCCs has been referred as a significant predictor of synchronous lesions: the highest risk is regarding the hypopharynx; regarding the oropharynx, the risk is intermediate; and the oral cavity and larynx are the locations with the lowest risk. ${ }^{1,6,12-15}$ Young age and limited initial tumors were considered as significant predictors of a synchronous esophageal tumor. ${ }^{1,6}$

In the United Kingdom, recent guidelines for tumor assessment and staging of HNSCCs recommend panendoscopy for patients with digestive symptoms, primary tumors with risk of a second synchronous primary tumor, ${ }^{16}$ or for the investigation of unknown primary tumor with metastatic neck HNSCC. ${ }^{17}$

In 2012, the French Society of Digestive Endoscopy established new guidelines that recommend flexible esophagoscopy with white light and targeted biopsies for every patient with a hypopharyngeal or oropharyngeal squamous cell carcinoma (SCC) or chronic alcohol intoxication.

However, in a cohort of 1,095 asymptomatic patients with high risk of developing esophageal carcinoma, Dubuc et al. ${ }^{18}$ reported $3.2 \%$ of cases of esophageal neoplasia and $5.4 \%$ in patients known with an HNSCC associated. The absence of symptoms or location of a primary tumor do not seem sufficient criteria to exclude a synchronous esophageal tumor. An esophageal examination should be performed for every patient with chronic alcohol consumption. For nondrinking patients, lack of information on the risk of a second location does not authorize going without an esophagoscopy.

Besides, synchronous tumors are likely to occur in the middle and lower esophagus, as they are in the cervical esophagus. ${ }^{6}$ The esophagus should be inspected in its entirety in every patient.

We propose optimizing the classic Savary procedure of panendoscopy: the optimized PLS provides a good-quality examination of the entire hypopharynx and upper esophageal sphincter. Flexible esophagoscopy with NBI provides the same quality of assessment of the esophagus. It is easier, faster, and safer. Likewise, a previous PLS examination of the glottic area enables the simplification of the bronchoscopy with a flexible bronchoscope.

The utility of rigid esophagoscopy could be reconsidered. Further studies should be conducted and compare the diag- nosis of a second primary tumor with rigid esophagoscopy versus the combination of PLS and flexible esophagoscopy prospectively.

\section{Conclusion}

Currently, the most sensitive method to diagnose early synchronous neoplasia in patients with a new HNSCC is a complete and systematic endoscopic examination.

This optimized PLS technique is easy, quick, affordable, effective, reliable, safe, and repeatable. In association with flexible esophagoscopy, it provides a good examination of the upper digestive tract. In case of complicated exposition, it can replace rigid esophagoscopy without loss in the evaluation.

\section{Presentations}

This study was presented in three congress:

- June 15, 2017: Luzern (Switzerland) - Spring Meeting of the HNS Swiss Society.

- October 08, 2017: Barcelona (Spain) - 4th Congress of European ORL-HNS.

- November 17, 2017: Poitiers (France) - 50th congress of the HNS French Society.

Conflict of Interests

The authors have no conflict of interests to declare.

\section{References}

1 McGarey PO Jr, O'Rourke AK, Owen SR, et al. Rigid Esophagoscopy for Head and Neck Cancer Staging and the Incidence of Synchronous Esophageal Malignant Neoplasms. JAMA Otolaryngol Head Neck Surg 2016;142(01):40-45

2 Koo K, Harris R, Wiesenfeld D, Iseli TA. A role for panendoscopy? Second primary tumour in early stage squamous cell carcinoma of the oral tongue. J Laryngol Otol 2015;129(Suppl 1):S27-S31

3 Wang WL, Lee CT, Lee YC, et al. Risk factors for developing synchronous esophageal neoplasia in patients with head and neck cancer. Head Neck 2011;33(01):77-81

4 Vergez S, Morinière S, Dubrulle FBilan initial des carcinomes épidermoïdes de la cavité buccale, du larynx et du pharynx (cavum exclu) Partie I: bilan d'extension locorégional, recommandations de la SFORL 2012, Annales françaises d'Oto-rhinolaryngologie et de Pathologie Cervico-faciale $130, \mathrm{n}^{\circ} 1$ (février. 2013:40-46

5 Lao-Sirieix P, Fitzgerald RC. Screening for oesophageal cancer. Nat Rev Clin Oncol 2012;9(05):278-287

6 Wexler SJ, Wernick B, Soliman AMS. Use of flexible esophagoscopy by otolaryngologists. Ann Otol Rhinol Laryngol 2014;123 (01):5-10

7 Rennemo E, Zätterström U, Boysen M. Impact of second primary tumors on survival in head and neck cancer: an analysis of 2,063 cases. Laryngoscope 2008;118(08):1350-1356

8 Tsao GJ, Damrose EJ. Complications of esophagoscopy in an academic training program. Otolaryngol Head Neck Surg 2010; 142(04):500-504

9 Cuellar SL, Carter BW, Macapinlac HA, et al. Clinical staging of patients with early esophageal adenocarcinoma: does FDG-PET/CT have a role? J Thorac Oncol 2014;9(08):1202-1206

10 Glaws WR, Etzkorn KP, Wenig BL, Zulfiqar H, Wiley TE, Watkins JL. Comparison of rigid and flexible esophagoscopy in the diagnosis of esophageal disease: diagnostic accuracy, complications, and cost. Ann Otol Rhinol Laryngol 1996;105(04):262-266 
11 Tsao GJ, Damrose EJ. Complications of esophagoscopy in an academic training program. Otolaryngol Head Neck Surg 2010; 142(04):500-504

12 de Monès E, Bertolus C, Salaun PY, et al; Socéité Française de l'Otorhinolaryngologie. Initial staging of squamous cell carcinoma of the oral cavity, larynx and pharynx (excluding nasopharynx). Part 2: Remote extension assessment and exploration for secondary synchronous locations outside of the upper aerodigestive tract. 2012 SFORL guidelines. Eur Ann Otorhinolaryngol Head Neck Dis 2013;130(02): 107-112

13 Haughey BH, Gates GA, Arfken CL, Harvey J. Meta-analysis of second malignant tumors in head and neck cancer: the case for an endoscopic screening protocol. Ann Otol Rhinol Laryngol 1992;101(2 Pt 1):105-112

14 Hung S-H, Tsai M-C, Liu T-C, Lin H-C, Chung S-D. Routine endoscopy for esophageal cancer is suggestive for patients with oral, oropharyngeal and hypopharyngeal cancer. PLoS One 2013;8 (08):e72097

15 Morimoto M, Nishiyama K, Nakamura S, et al. Significance of endoscopic screening and endoscopic resection for esophageal cancer in patients with hypopharyngeal cancer. Jpn J Clin Oncol 2010;40(10):938-943

16 Roland N, Porter G, Fish B, Makura Z. Tumour assessment and staging: United Kingdom National Multidisciplinary Guidelines. J Laryngol Otol 2016;130(S2):S53-S58

17 Mackenzie K, Watson M, Jankowska P, Bhide S, Simo R. Investigation and management of the unknown primary with metastatic neck disease: United Kingdom National Multidisciplinary Guidelines. J Laryngol Otol 2016;130(S2):S170-S175

18 Dubuc J, Legoux J, Winnock M, et al; Société Française d'Endoscopie Digestive. Endoscopic screening for esophageal squamous-cell carcinoma in high-risk patients: a prospective study conducted in 62 French endoscopy centers. Endoscopy 2006;38(07):690-695 